\title{
The tumor suppressor Wnt inhibitory factor 1 is frequently methylated in nasopharyngeal and esophageal carcinomas
}

\author{
Stephen L Chan ${ }^{1, *}$, Yan Cui ${ }^{1, *}$, Andrew van Hasselt ${ }^{2}$, Hongyu Li ${ }^{1}$, Gopesh Srivastava ${ }^{3}$, Hongchuan Jin ${ }^{1}, \mathrm{Ka} \mathrm{M} \mathrm{Ng}^{1}$, \\ Yajun Wang ${ }^{1}$, Kwan Y Lee ${ }^{1}$, George SW Tsao ${ }^{4}$, Sheng Zhong ${ }^{5}$, Keith D Robertson ${ }^{5}$, Sun Y Rha ${ }^{6}$, Anthony TC Chan ${ }^{1}$ \\ and Qian Tao ${ }^{1,7}$
}

Aberrant activation of the wingless-type- (Wnt)-signaling pathway is common in many cancers including nasopharyngeal (NPC) and esophageal squamous cell (ESCC) carcinomas, both prevalent in Southern China and Southeast Asia. However, the molecular mechanism leading to this abnormality is still obscure. Wnt inhibitory factor-1 (WIF1) is a secreted antagonist of the Wnt pathway, and is recently shown to be inactivated by epigenetic mechanism in some tumors. Here, we examined whether WIF1 is also inactivated epigenetically in NPC and ESCC. With semiquantitative reverse transcriptionPCR and methylation-specific PCR, we detected WIF1 downregulation or silencing in 6/6 of NPC and 12/19 of ESCC cell lines, which is well correlated with its methylation status. Methylation was further confirmed by high-resolution bisulfite genomic sequencing. Methylation was also frequently observed in a large collection of primary tumors of NPC $(85 \%, 55 /$ $65)$ and ESCC (27\%, 25/92), with WIF1 expressed and unmethylated in normal NPC and esophageal cell lines and normal tissues. Treatment of 5-aza-2'-deoxycytidine demethylated WIF1 and induced its expression in NPC and ESCC cell lines, highlighting a direct role of epigenetic inactivation. Ectopic expression of WIF1 in NPC and ESCC tumor cells resulted in significant inhibition of tumor cell colony formation, similar to TP53, and also significant downregulation of $\beta$-catenin protein level in NPC cells. Thus, WIF1 functions as a tumor suppressor for both NPC and ESCC through suppressing the Wnt-signaling pathway, but is frequently silenced by epigenetic mechanism in a tumor-specific way. Our study indicates that epigenetic inactivation of WIF1 contributes to the aberrant activation of Wnt pathway and is involved in the pathogenesis of both tumors. WIF1 methylation could also serve as a specific biomarker for these tumors.

Laboratory Investigation (2007) 87, 644-650; doi:10.1038/labinvest.3700547; published online 26 March 2007

KEYWORDS: WIF1; tumor suppressor gene; methylation; $\beta$-catenin; nasopharyngeal carcinoma; esophageal carcinoma

Wingless-type (Wnt) protein family is a group of secreted glycoproteins, which plays a pivotal role in regulation of proliferation and differentiation of epithelial cells. ${ }^{1-3}$ The associated canonical Wnt-signaling pathway (known as the wingless pathway in Drosophila) is the most intensively studied Wnt pathway implicated in oncogenesis. The pathway involves binding of Wnt ligands to the Frizzled family receptors at the cell surface which leads to activation of intracellular molecules disheveled. Disheveled in turn inhibits the glycogen synthase kinase $3 \beta(\mathrm{GSK} 3 \beta)$ and allows cytoplasmic accumulation of $\beta$-catenin. ${ }^{4,5}$ As a result, $\beta$-catenin translocates into nucleus, interacts with T-cell factor/lymphocyte enhancer binding factor, ${ }^{6,7}$ and activates transcription of target oncogenes such as cyclin D1, c-Myc and $V E G F .^{6,8-11}$

Two groups of Wnt antagonist with different mechanisms of action have been identified. ${ }^{12}$ One group (Dickkopf family) inhibits the Wnt signaling by binding to the LRP5/LRP6

\footnotetext{
${ }^{1}$ Cancer Epigenetics Laboratory, State Key Laboratory in Oncology in South China, Department of Clinical Oncology, Sir YK Pao Center for Cancer, Hong Kong; ${ }^{2}$ Department of Surgery, Hong Kong Cancer Institute and Li Ka Shing Institute of Health Sciences, The Chinese University of Hong Kong, Hong Kong; ${ }^{3}$ Department of Pathology, The University of Hong Kong, Hong Kong; ${ }^{4}$ Department of Anatomy, The University of Hong Kong, Hong Kong; ${ }^{5}$ Department of Biochemistry and Molecular Biology, University of Florida, FL, USA; ${ }^{6}$ Yonsei Cancer Center, Yonsei University College of Medicine, Seoul, Korea and ${ }^{7}$ Johns Hopkins Singapore and Sidney Kimmel Comprehensive Cancer Center, Johns Hopkins School of Medicine, Baltimore, MD, USA

Correspondence: Dr Q Tao, PhD, Rm315, Cancer Center, Department of Clinical Oncology, PWH, The Chinese University of Hong Kong, Shatin, Hong Kong.

E-mail: qtao@clo.cuhk.edu.hk

*These authors contributed equally to this work.

Received 07 July 2006; revised 28 January 2007; accepted 29 January 2007
} 
subunit of Wnt receptor complex. Wnt inhibitory factor 1 (WIF1) belongs to another group, which acts by direct binding to Wnt ligands. WIF1 is composed of an N-terminal signal peptide sequence and 5-epidermal growth factor like repeats. The WIF1 gene, located at 12q14, was first identified from human retina. ${ }^{13}$ It is a highly conserved gene, and does not share any sequence similarities with the cystine-rich domains of Frizzled or secreted frizzled-related protein. There are dense CpG sites in the WIF1 promoter (Figure 1a), and methylation of these $\mathrm{CpG}$ sites leads to the inactivation of WIF1 in tumors. WIF1 was found to be downregulated by methylation in various human malignancies including carcinoma of lungs, mesothelioma, colorectal, various urological and gastrointestinal malignancies. ${ }^{14-16}$ We also reported that WIF1 is frequently inactivated by methylation in breast cancer. ${ }^{17}$

Compared with the low incidence in other parts of the world, nasopharyngeal carcinoma (NPC) and esophageal squamous cell carcinoma (ESCC) are both prevalent malignancies in Southern Asia including Southern China such as Hong Kong, with annual incidence reaching $\sim 30$ and $\sim 100$ / 100000 , respectively. Despite high prevalence of both tumors, their molecular pathogenesis, especially regarding the epigenetic aspect, is still poorly known. An increasing amount of evidence shows that the $\mathrm{Wnt} / \beta$-catenin pathway is aberrantly activated in both NPC and ESCC. ${ }^{18,19}$ However, the detailed components of Wnt pathway responsible for this aberrant activation is still to be elucidated. In this study, we examined the epigenetic alteration and the tumor suppressor function of WIF1, a recently identified antagonist of the Wnt $/ \beta$ catenin pathway, in NPC and ESCC.

\section{MATERIALS AND METHODS}

\section{Cell Lines, Tumor Samples and Drug Treatment}

A panel of tumor cell lines used, including NPC (C666-1, CNE1, CNE2, HK1, HNE1 and HONE1), and ESCC (EC1,
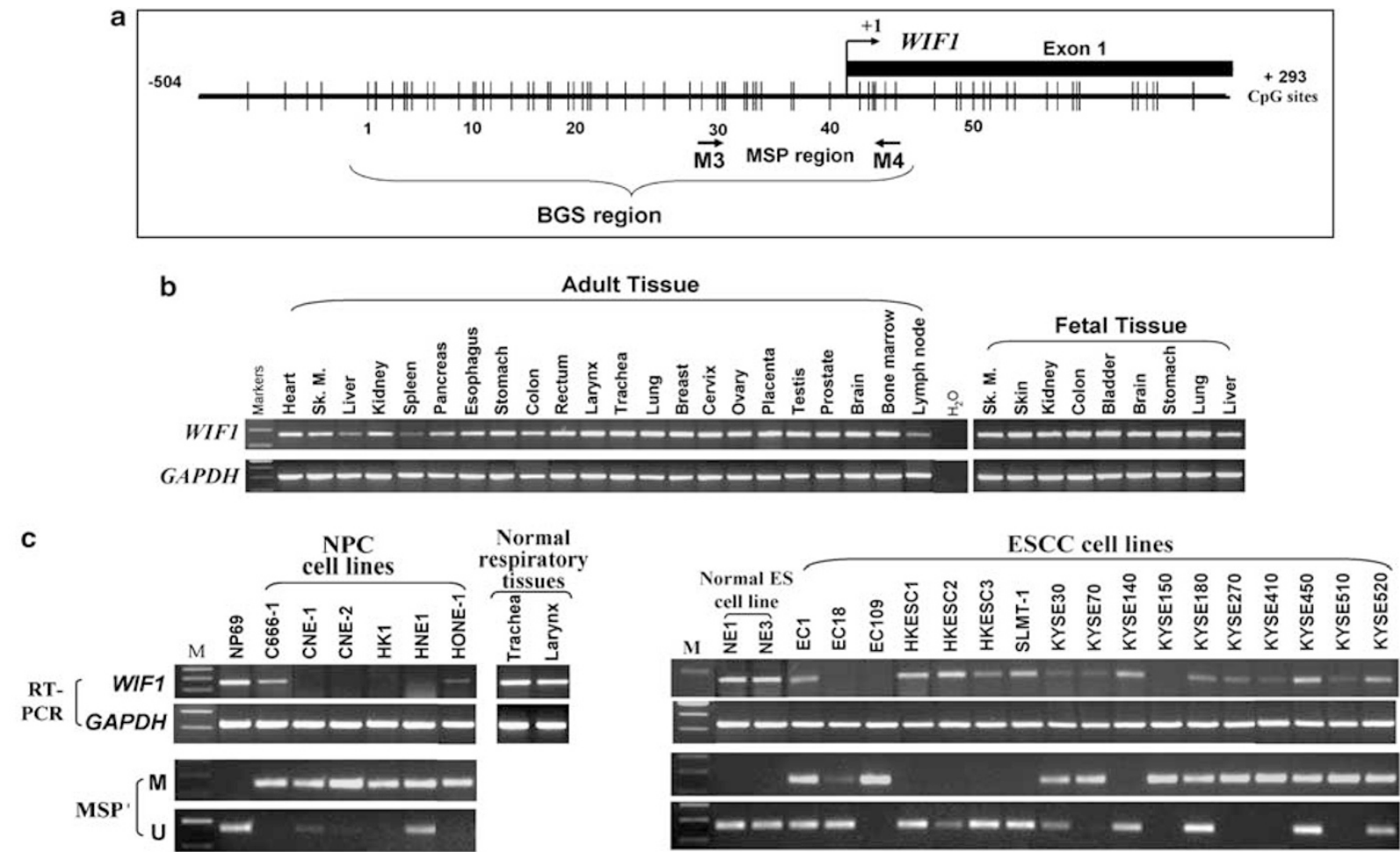

d

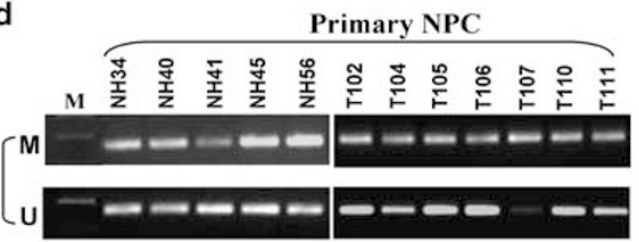

e

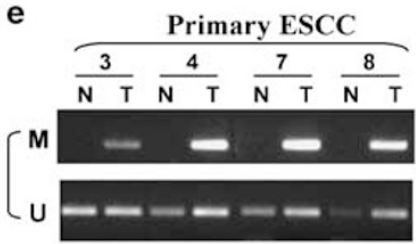

f

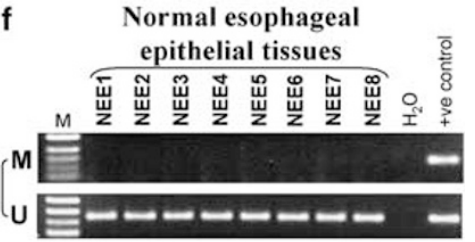

Figure 1 (a) Schematic structure of the WIF1 promoter CGI. The exon 1 is indicated with a dark rectangle, CpG sites in the CGI are indicted by short vertical lines, and the transcription start site indicated by a curved arrow. The MSP and BGS regions analyzed are also indicated. M3 and M4 represent MSP primers. (b) Broad expression of WIF1 in human normal adult and fetal tissues. (c) Silencing of WIF1 by promoter methylation in NPC and ESCC cell lines, but not in normal epithelial cell lines. M, methylation; $U$, unmethylation. (d) Representative MSP results of NPC primary tumors. (e) Representative MSP results of ESCC primary tumors and paired normal tissues. No methylation was detected in paired normal sample $(\mathrm{N})$ whereas methylation was frequently observed in ESCC tumors (T). (f) MSP results of NEE, with no methylation detected. M, molecular markers. 
EC18, EC109, HKESC1, HKESC2, HKESC3, SLMT-1, KYSE30, KYSE70, KYSE140, KYSE150, KYSE180, KYSE270, KYSE410, KYSE450, KYSE510, KYSE520, KYSE50 and KYSE220). Three immortalized normal epithelial cell lines, including one nasopharyngeal (NP69) and two esophageal (ES) (NE1, NE3) with many features of normal epithelial cells were also used as controls. ${ }^{20-22}$ These cell lines were routinely maintained in RPMI1640 or DMEM medium. Total RNA and DNA were extracted from cell pellets or tumor tissues using TriReagent. ${ }^{23}$ Source of DNA samples of NPC and ESCC tumors have been described previously. ${ }^{21-24}$ Eight normal esophageal epithelial tissues (NEE) were collected in the Department of Pathology, University of Hong Kong. ${ }^{25}$ For drug treatment, cell lines were treated with 5-aza- $2^{\prime}$ deoxycytidine (Aza) (Sigma, ST Louis, MO, USA; $50 \mu \mathrm{M}$ for NPC cell lines and $10 \mu \mathrm{M}$ for ESCC cell lines) for 3 days, as described previously. ${ }^{23}$

\section{Reverse Transcription-PCR}

Semi-quantitative reverse transcription (RT)-PCR was performed as previously described using Taq-Gold, with $G A P D H$ as an internal control. ${ }^{23}$ In brief, RT-PCR was performed by using AmpliTaq Gold (Applied Biosystems, Foster City, CA, USA), and the PCR programs started with an initial denaturation at $95^{\circ} \mathrm{C}$ for $10 \mathrm{~min}$, followed by 37 cycles $\left(94^{\circ} \mathrm{C}\right.$ for $30 \mathrm{~s}, 55^{\circ} \mathrm{C}$ for $30 \mathrm{~s}$ and $72^{\circ} \mathrm{C}$ for $30 \mathrm{~s}$ ) of amplification, with a final extension at $72^{\circ} \mathrm{C}$ for $10 \mathrm{~min}$. PCR bands were visualized under UV light and photographed. Primers used were WIF1F: 5'-TATGGATCGATGCTCACCAG and WIF1R: 5'-CAGAGGGACATTGACGGTTG.

\section{Methylation-Specific PCR and Bisulfite Genomic Sequencing}

Bisulfite modification of DNA was carried out as previously described. ${ }^{26}$ In brief, $5 \mu \mathrm{g}$ of genomic DNA was denatured by adding $3 \mu \mathrm{l}$ of $3 \mathrm{M} \mathrm{NaOH}$ at $37^{\circ} \mathrm{C}$ for $15 \mathrm{~min}$. Denatured DNA was mixed with $333 \mu \mathrm{l}$ of bisulfite solution and incubated in darkness for $4 \mathrm{~h}$ at $55^{\circ} \mathrm{C}$. The bisulfite solution was prepared as $2.4 \mathrm{M}$ sodium metabisulfite ( $\mathrm{pH}$ 5.0-5.2) (Sigma) $/ 0.5 \mathrm{mM}$ hydroquinone. Treated DNA was desalted and purified using the QIAEX II kit (Qiagen). DNA was then treated with $0.3 \mathrm{M} \mathrm{NaOH}$ at $37^{\circ} \mathrm{C}$ for $15 \mathrm{~min}$ and purified with QIAEX II. Recovered DNA was eluted in $100 \mu \mathrm{l}$ of TE buffer $(\mathrm{pH} 8.0)$ and stored at $-20^{\circ} \mathrm{C}$. For methylation-specific PCR (MSP), bisulfite-modified DNA was amplified using either a methylation-specific or unmethylation-specific primer set. ${ }^{27}$ Methylation-specific primers were WIF1M3: 5'-TGTCGTTTTTATTTTCGTTCGC and WIF1M4: 5'-CGTT TAAACGACTAAACGCG, whereas the unmethylation-specific primers were WIF1U3: $5^{\prime}$-TTTTTGTTGTTTTTATTTTT GTTTGT and WIF1U4: 5'-TCCCATTTAAACAACTAAAC ACA. MSP was performed for 40 (for methylation-specific primers; $60^{\circ} \mathrm{C}$ ) or 42 (for unmethylation-specific primers; $60^{\circ} \mathrm{C}$ ) cycles using AmpliTaq Gold and hot-start. ${ }^{27}$ MSP primers were tested and confirmed not to amplify any unbisulfited DNA. For bisulfite genomic sequencing (BGS) to analyze the methylation status of $46 \mathrm{CpG}$ sites in a 463-bp region of the WIF1 promoter $(-402$ to +61$)$, bisulfite-treated DNA was amplified using a BGS primer set (WIF1BGS1: 5'-GTTTTAGGGGTTTTTGAGTGTT; WIF1BGS2: 5'-CAACT CCCTCAACCAAAACTA), and the PCR products were cloned into pCR4-Topo vector (Invitrogen, Carlsbad, CA, USA). ${ }^{26}$ Around eight colonies were randomly chosen and sequenced.

\section{SDS-PAGE and Western Blotting}

Anti- $\alpha$-tubulin (DM1A, Neomarkers), Anti- $\beta$-catenin (M3539, Dako) and HRP-coupled rabbit anti-mouse secondary antibody (P0161, Dako) were used. Lysates of cells transfected with pcDNA3.1 or pcDNA3.1-WIF1 were separated on $10 \%$ Bis/Tris-polyacrylamide gel through electrophoresis and blotted onto nitrocellulose membranes (Amersham). Blots were immunostained with primary antibodies overnight at $4{ }^{\circ} \mathrm{C}$ and secondary antibody for $1 \mathrm{~h}$ at room temperature. Proteins were visualized using ECL Plus Western blotting Detection Reagents (RPN2132, GE Healthcare). The film was scanned with a GS-700 Densitometer, and quantitated by Quantity One software (Bio-Rad).

\section{Colony Formation Assay using Monolayer Culture}

The full-length cDNA of WIF1 was PCR cloned, sequenceverified, and further subcloned into the expression vector pcDNA3.1(-) (Invitrogen, Carlsbad, CA, USA) through the XhoI-HindIII sites. For monolayer culture, freshly seeded tumor cells $\left(1 \times 10^{5} /\right.$ well $)$ plated in a 12 -well plate were cultured for overnight, then transfected with various expression plasmids or the empty vector $(0.5 \mu \mathrm{g}$ each) using FuGENE 6 (Roche). ${ }^{21,22}$ Cells were plated in a 6 -well plate $48 \mathrm{~h}$ post-transfection, and selected for 1-2 weeks with G418. Untransfected cells could not survive with G418 selection and surviving colonies were stained with Gentian Violet. The experiments were performed in triplicate wells for three times. The TP53-expressing plasmid (gift of Dr Bert Vogelstein) was used as a positive control. ${ }^{22}$ The results were shown as values of mean \pm s.e. Statistical analysis was carried out with Student's $t$-test, and $P<0.05$ was considered as statistically significant difference.

\section{RESULTS \\ Broad Expression of WIF1 in Normal Tissues but Frequent WIF1 Silencing by Methylation in NPC and ESCC Cell Lines}

By semiquantitative RT-PCR with GAPDH as a control, the expression of WIF1 was evaluated in a panel of human normal tissues and tumor cell lines. WIF1 expression was readily detectable in all normal adult and fetal tissues, although with varied expression levels (Figure 1b), including normal upper respiratory tract tissues (larynx, trachea) and esophagus, which are the normal counterparts for NPC and esophageal carcinoma. In contrast, WIF1 expression was lost 
or considerably reduced in NPC $(6 / 6,100 \%)$ and ESCC (12/ 19, 63\%) cell lines (Figure 1c).

To test whether WIF1 silencing is a consequence of epigenetic inactivation, the methylation status of the WIF1 promoter was analyzed by both MSP and BGS. For MSP, methylated alleles were detected in those cell lines with absent or reduced expression, whereas no methylation was observed in normal esophageal (NE1, NE3) and nasopharyngeal epithelial cell lines (NP 69) (Figure 1c). Methylation was not detected in most of the WIF1-expressing cell lines; however, for some expressing cell lines both methylated and unmethylated alleles were detected. The MSP results were confirmed by BGS analyses of a 463-bp region of the WIF1 promoter, which demonstrated densely methylated $\mathrm{CpG}$ sites at the WIF1 promoter in silenced cell lines but not in normal epithelial cell lines (Figure 2).

\section{Tumor-Specific Methylation of WIF1 in Primary NPC and ESCC}

We further examined WIF1 methylation in a large collection of primary tumors, some with paired normal tissues. Methylation was observed in 55 of 65 (85\%) NPC (Figure 1d) and 25 of 92 (27\%) ESCC tumor samples (Figure 1e). For ESCC, paired non-tumorous tissues were available for analysis, of which no methylation was observed in all the 92 samples. Furthermore, all the eight normal esophageal tissue samples had no methylation at all (Figure 1f). Detailed methylation analysis using BGS revealed heavily methylated alleles together with unmethylated alleles (which represent the tumor-infiltrating normal cells) in primary tumors (Figure 2). On the other hand, only few methylated CpG sites were detected in paired normal esophageal tissues (Figure 2).

\section{Pharmacologic Demethylation of the WIF1 Promoter Restores its Expression in Tumor Cell Lines}

Treatment of both NPC and ESCC cell lines with 5-aza-2'deoxycytidine (Aza) induced demethylation of the WIF1 promoter and its concomitant expression (Figure 3a). Both MSP and BGS confirmed the demethylation of the promoter in individual alleles, or at individual CpG sites (Figure 3b).

\section{WIF1 Functions as a Tumor Suppressor through Downregulating $\beta$-Catennin Protein Level}

The frequent silencing of WIF1 in both NPC and ESCC but not normal epithelial tissues or cell lines suggests that WIF1 probably functions as a tumor suppressor in both tumors. To test this hypothesis, colony formation assay was used to analyze the tumor suppressor effect of WIF1. We chose CNE2, HONE1 (NPC cell lines) and EC109 (ESCC cell line) for transfection owing to their silencing of WIF1 and promoter methylation. The colony formation efficiencies of these cell lines were significantly suppressed after transfection with WIF1, comparable to that observed in TP53-transfected cells (Figure 4a and b), indicating that WIF1 functions as a tumor suppressor in both NPC and ESCC cells.
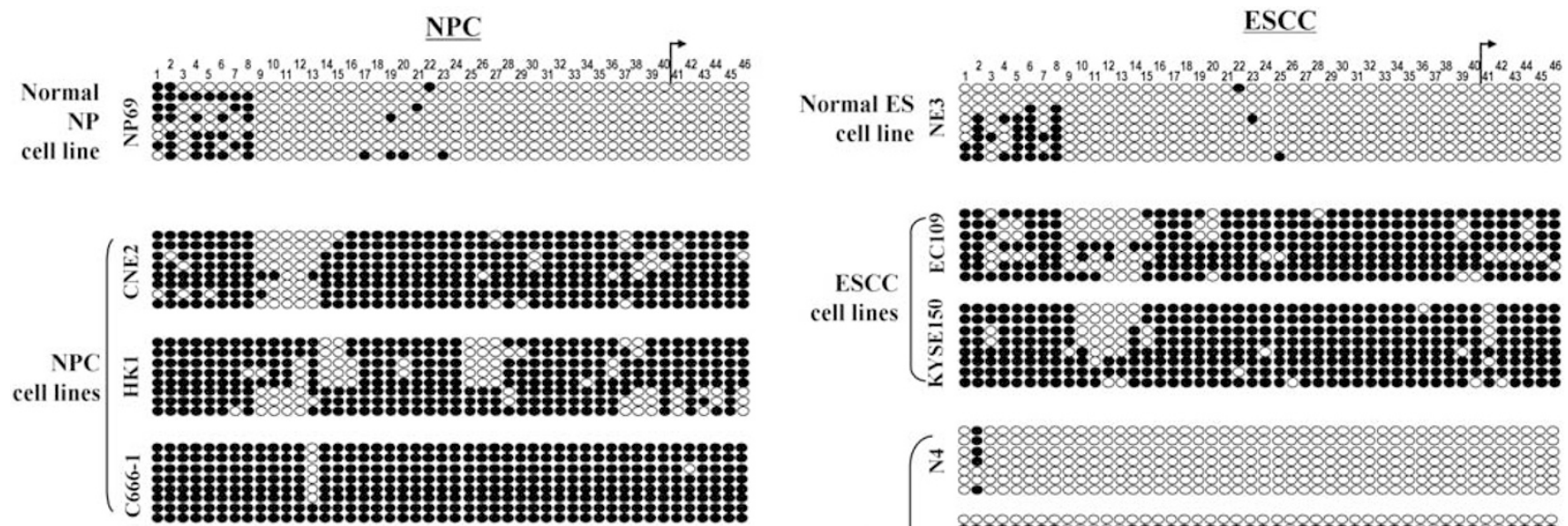

ESCC cell lines
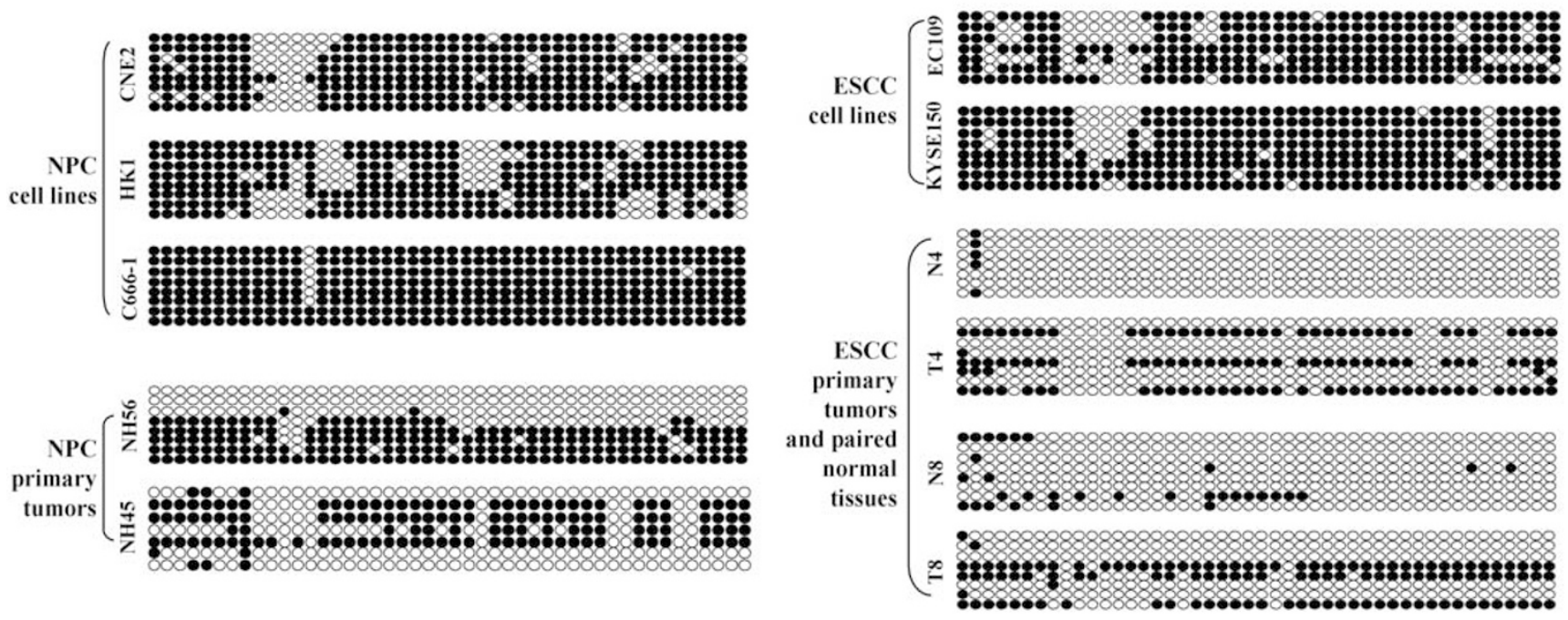

Figure 2 Representative high-resolution analyses of the methylation status of individual CpG sites in the WIF1 promoter by BGS in NPC and ESCC cell lines and primary tumors. Each row indicates a promoter allele analyzed and each circle corresponds to a single CpG site. Methylated sites are indicated as filled dark circles while unmethylated sites as empty white ones. NP, nasopharyngeal epithelium; ES, esophageal epithelium. 
a

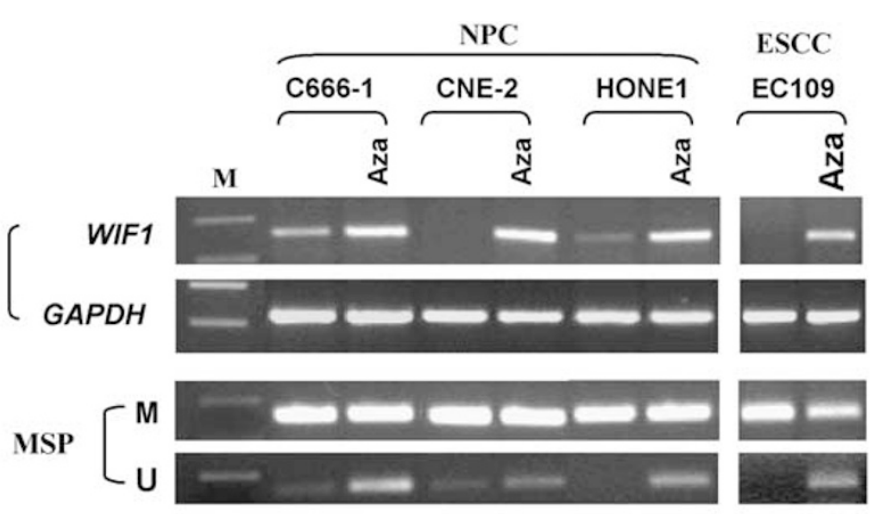

b

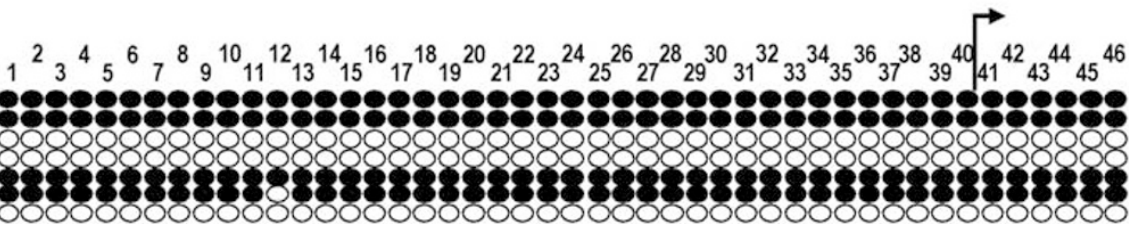

Figure 3 (a) Pharmacologic demethylation and reactivation of WIF1 with 5-aza-2'-deoxycytidine (Aza) treatment in NPC and ESCC cells. Semiquantitative RT-PCR analysis detected upregulated expression and MSP detected concomitant demethylation of the promoter in Aza-treated cell lines. (b) Detailed BGS analysis showed that the percentage of methylated CpG sites was significantly reduced after treatment with Aza.

We further determined the molecular mechanism of this tumor suppression. As $\beta$-catenin is accumulated in both NPC and ESCC, WIF1 could exert its tumor suppressor functions through modulating $\beta$-catenin level. Thus, intracellular protein level of $\beta$-catenin in HONE1 before and after ectopic WIF1 expression was determined by Western blot. Indeed, the intracellular $\beta$-catenin protein level was significantly reduced after WIF1 expression (Figure 4c). Thus, WIF1 functions as a tumor suppressor in NPC through suppressing the Wnt-signaling pathway.

\section{DISCUSSION}

In the present study, we demonstrated that WIF1 is silenced by epigenetic mechanism in the majority of cell lines and large numbers of primary tumors of NPC and ESCC. This is consistent with studies in other cancer types, which reported promoter methylation as the major mechanism for inactivation of this tumor suppressor gene. Furthermore, using transfection of cell lines with WIF1-expressing plasmid, we demonstrated that ectopic expression of WIF1 in both NPC and ESCC cell lines could significantly reduce the colony formation of tumor cells, highlighting the fact that WIF1 is a functional tumor suppressor in these two types of tumors. We also showed evidence that WIF1 exerts its tumor suppressor functions through downregulating intracellular protein level of $\beta$-catenin thus suppressing the Wnt-signaling pathway.

Cancer development depends on the aberrant deregulation of signal transduction pathways that control cell growth and survival. Canonical Wnt signaling pathway is an important pathway involved in tumor pathogenesis. ${ }^{1}$ As the initial report of aberrant activation of Wnt pathway in colorectal carcinoma, Wnt pathway had been linked up with multiple other cancer types. ${ }^{7,28}$ Meanwhile, epigenetic gene silencing is associated with the onset and progression of various cancers, and such events can even precede genetic changes during carcinogenesis. ${ }^{29}$ Epigenetic modification can be achieved via either promoter methylation and/or modification of histone tails in the chromatin. These two epigenetic events are closely linked and affect each other through interactions between DNA methyltransferase and histone deacetylase complex at methylated $\mathrm{CpG}$ sites. ${ }^{30,31}$ Recently, pharmacologic demethylation has been explored as a cancer therapeutic strategy through restoring tumor suppressor gene functions which would induce tumor cell apoptosis or reactivate silenced viral immunodominant proteins. ${ }^{32,33}$

NPC is a prevalent tumor in Southern China and Southeast Asia, especially in the 20-44 age group. Despite effective primary treatment, NPC remains to cause a lot of morbidity and mortality because of its high propensity of distant metastasis. Although $\beta$-catenin was found to be accumulated in $\mathrm{NPC},{ }^{34}$ studies on Wnt pathway genes in NPC pathogenesis are still scanty. In one study with cDNA microarray expression profiling, the Wnt pathway was found to be deregulated, whereas its inhibitory molecule AXIN2 was downregulated in NPC. ${ }^{19}$ Our results go in line with the above findings and suggest that epigenetic silencing of another antagonist of the Wnt pathway, WIF1, contributes to the pathogenesis of NPC. Previous works from our team had shown that demethylating agents, azacitidine, can induce in vivo reversal of $\mathrm{CpG}$ islands (CGI) methylation of Epstein-Barr virus (EBV) genes in NPC. ${ }^{32}$ Although it is still unclear how significant the role of WIF1 silencing is in inducing aberrant activation of Wnt pathway in vivo, in view of its high frequency of epigenetic 
a
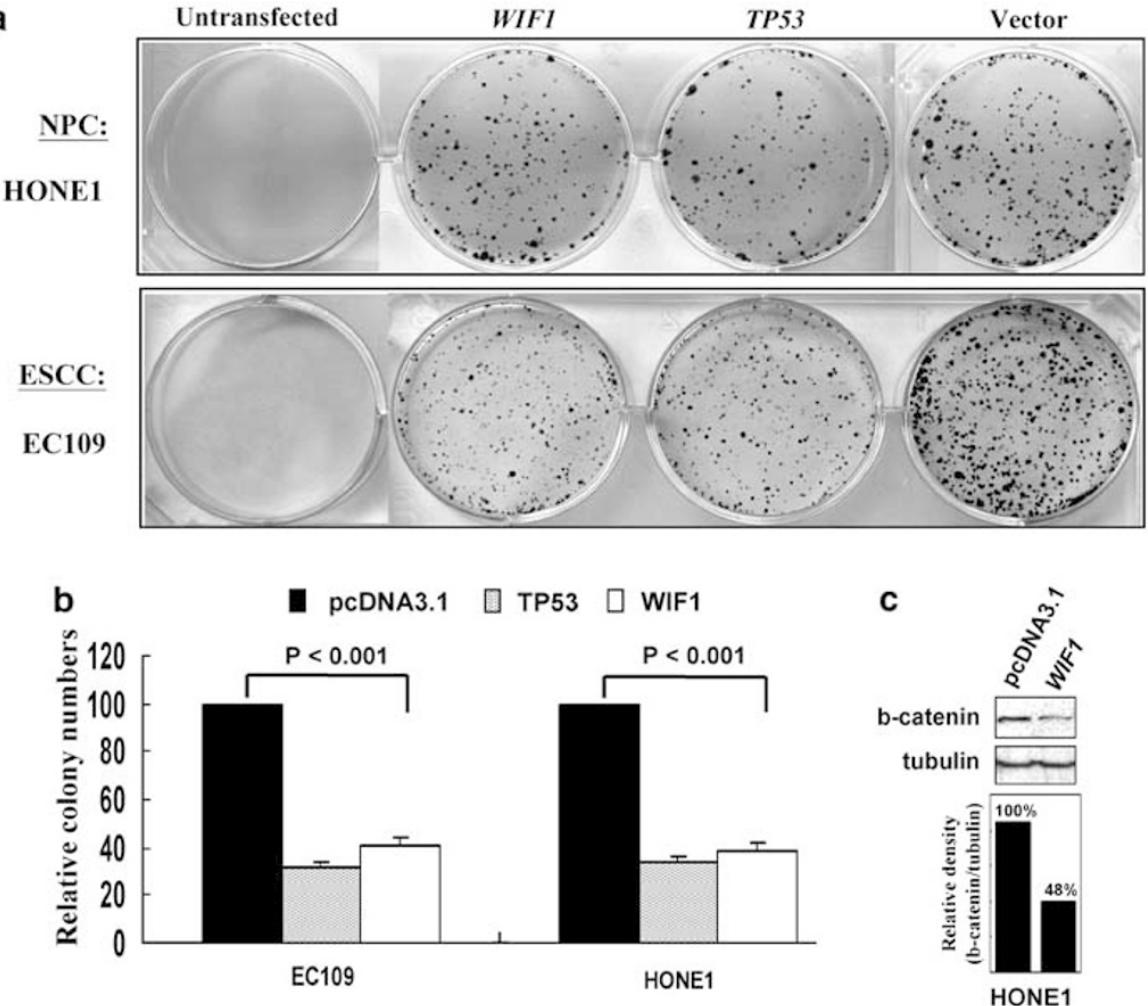

Figure 4 Assessing the tumor suppressor function of WIF1 in NPC (HONE1) and ESCC (EC109) cells using colony formation assays (monolayer culture). (a) Colony formation efficiencies were significantly inhibited in WIF1-expressing cells, which was similar to cells transfected with a TP53-expressing plasmid, when compared with control cells transfected with vector only. (b) Quantitative analyses of the results in (a). (c) WIF1 functions as a tumor suppressor through downregulating $\beta$-catenin protein level. Intracellular $\beta$-catenin level before and after ectopic WIF1 expression was determined by Western blot with monoclonal anti- $\beta$-catenin antibody (upper panel), with $\alpha$-tubulin as a loading control (middle panel). The signals were further quantitated (bottom panel).

inactivation in NPC cell lines and primary tumors, WIF1 could be one of the ideal targets for future development of epigenetic therapeutics.

ESCC remains to be one of the most lethal gastrointestinal malignancies in China and other parts of the world. It is well known that the Wnt pathway is aberrantly activated in ESCC, although the mechanism of this abnormality is poorly understood. ${ }^{35,36}$ In a recent study, WIF1 was found to be silenced epigenetically in a limited number of Japanese ESCC cell lines and primary tumors. ${ }^{15}$ We have systematically analyzed a larger series of ESCC cell lines and a much larger number of primary tumors from Hong Kong Chinese as well as paired normal esophageal tissues, NEE tissues and normal cell lines. The results from these two ethnic groups were similar. We found that WIF1 was only inactivated in ESCC cell lines and tumors, whereas readily expressed in NEE cell lines. No methylation of WIF1 was detected in normal esophageal cell lines and paired normal tissues. We also noticed that in rare cell lines like EC18, WIF1 expression was low or silent but without promoter methylation, indicating that other regulatory mechanism such as histone remodeling is probably also infrequently involved in silencing WIF1 in tumors. Although question remains whether accumulation of $\beta$ catenin in cytosol and nucleus would render ESCC patients poorer prognosis, it had been shown that esophageal tumor cell growth could be inhibited by downregulation of $\beta$-catenin with antisense oligonucleotides. ${ }^{37}$ It is therefore of interest to explore further whether demethylation therapy activating WIF1 will reduce the intracellular $\beta$-catenin level and suppress tumor cell growth.

The $\mathrm{Wnt} / \beta$-catenin cascade has various components which ranges from Wnt ligands, Frizzled, disheveled, AXIN/APC/ GSK complexes to $\beta$-catenin. One may reasonably wonder about the significance of those upstream events in affecting the whole Wnt pathway and the final effector $\beta$-catenin. In one of the report, restoration of WIF1 function induced significant apoptosis in colorectal cancer cell lines containing downstream mutations. ${ }^{6}$ This underscores the therapeutic value of reversing WIF1 methylation in suppressing the Wnt cascade despite the presence of downstream mutations, and indicates a possible Wnt pathway-independent mechanism of apoptosis induction by WIF1.

The clinical impact of WIF1 inactivation in both NPC and ESCC is unknown. Further studies of clinical correlation of WIF1 methylation in a large collection of NPC and ESCC patients are required to answer this question. It is of interest to notice that the histology of NPC in Asia is usually poorly differentiated, whereas ESCC is a more differentiated 
squamous cell carcinoma. Previous studies of other tumors such as prostate, breast and non-small cell lung carcinoma showed that loss of WIF1 expression was not significantly associated with tumor stage or grade, suggesting that epigenetic silencing of WIF1 might be an early event in carcinogenesis. ${ }^{16}$ Such characteristics may enable the detection of WIF1 methylation in NPC or ESCC tumor tissues or serum samples as a molecular marker for screening and diagnosis.

In conclusion, we have demonstrated that WIF1 is frequently epigenetically inactivated in NPC and ESCC, and WIF1 also functions as a tumor suppressor in both tumors through downregulating $\beta$-catenin protein level and suppressing the Wnt-signaling pathway.

\section{ACKNOWLEDGEMENT}

We thank Drs Kaitai Yao, Ya Cao, (Dolly Huang) and Guiyuan Li for some cell lines and Bert Vogelstein for the TP53 CDNA. This study was supported by a Hong Kong RGC Grant (CUHK4443/05M) and The Chinese University of Hong Kong.

1. Akiyama T. Wnt/beta-catenin signaling. Cytokine Growth Factor Rev 2000;11:273-282.

2. Seidensticker MJ, Behrens J. Biochemical interactions in the wnt pathway. Biochim Biophys Acta 2000;1495:168-182.

3. Yamaguchi TP. Heads or tails: Wnts and anterior-posterior patterning Curr Biol 2001;11:713-724.

4. Ikeda S, Kishida S, Yamamoto $\mathrm{H}$, et al. Axin, a negative regulator of the Wnt signaling pathway, forms a complex with GSK-3beta and betacatenin and promotes GSK-3beta-dependent phosphorylation of betacatenin. EMBO J 1998;17:1371-1384.

5. Ikeda S, Kishida M, Matsuura Y, et al. GSK-3beta-dependent phosphorylation of adenomatous polyposis coli gene product can be modulated by beta-catenin and protein phosphatase $2 \mathrm{~A}$ complexed with Axin. Oncogene 2000;19:537-545.

6. He TC, Sparks $A B$, Rago $C$, et al. Identification of c-MYC as a target of the APC pathway. Science 1998;281:1509-1512.

7. Morin PJ, Sparks $A B$, Korinek $V$, et al. Activation of beta-catenin-TCF signaling in colon cancer by mutations in beta-catenin or APC. Science 1997;275:1787-1790.

8. Gotoh J, Obata M, Yoshie M, et al. Cyclin D1 over-expression correlates with beta-catenin activation, but not with $\mathrm{H}$-ras mutations, and phosphorylation of Akt, GSK3 beta and ERK1/2 in mouse hepatic carcinogenesis. Carcinogenesis 2003;24:435-442.

9. Li YJ, Wei ZM, Meng YX, et al. Beta-catenin up-regulates the expression of cyclinD1, c-myc and MMP-7 in human pancreatic cancer: relationships with carcinogenesis and metastasis. World J Gastroenterol 2005;11:2117-2123.

10. Tetsu O, McCormick F. Beta-catenin regulates expression of cyclin D1 in colon carcinoma cells. Nature 1999;398:422-426.

11. Easwaran V, Lee $\mathrm{SH}$, Inge $\mathrm{L}$, et al. beta-Catenin regulates vascular endothelial growth factor expression in colon cancer. Cancer Res 2003:63:3145-3153.

12. Kawano Y, Kypta R. Secreted antagonists of the Wnt signalling pathway. J Cell Sci 2003;116:2627-2634.

13. Hsieh JC, Kodjabachian $\mathrm{L}$, Rebbert $\mathrm{ML}$, et al. A new secreted protein that binds to Wnt proteins and inhibits their activities. Nature 1999;398:431-436.

14. Mazieres J, He B, You L, et al. Wnt inhibitory factor-1 is silenced by promoter hypermethylation in human lung cancer. Cancer Res 2004:64:4717-4720.

15. Taniguchi $H$, Yamamoto $H$, Hirata $T$, et al. Frequent epigenetic inactivation of Wnt inhibitory factor-1 in human gastrointestinal cancers. Oncogene 2005;24:7946-7952.

16. Wissmann C, Wild PJ, Kaiser S, et al. WIF1, a component of the Wnt pathway, is down-regulated in prostate, breast, lung, and bladder cancer. J Pathol 2003;201:204-212.
17. Ai L, Tao Q, Zhong S, et al. Inactivation of Wnt inhibitory factor-1 (WIF1) expression by epigenetic silencing is a common event in breast cancer. Carcinogenesis 2006;27:1341-1348.

18. Nakajima M, Fukuchi M, Miyazaki T, et al. Reduced expression of Axin correlates with tumor progression of oesophageal squamous cell carcinoma. Br J Cancer 2003;88:1734-1739.

19. Sriuranpong V, Mutirangura A, Gillespie JW, et al. Global gene expression profile of nasopharyngeal carcinoma by laser capture microdissection and complementary DNA microarrays. Clin Cancer Res 2004; 10:4944-4958.

20. Tsao SW, Wang X, Liu Y, et al. Establishment of two immortalized nasopharyngeal epithelial cell lines using SV40 large T and HPV16E6/ E7 viral oncogenes. Biochim Biophys Acta 2002;1590:150-158.

21. Ying J, Li H, Seng TJ, et al. Functional epigenetics identifies a protocadherin $\mathrm{PCDH} 10$ as a candidate tumor suppressor for nasopharyngeal, esophageal and multiple other carcinomas with frequent methylation. Oncogene 2006;25:1070-1080.

22. Ying J, Srivastava G, Hsieh WS, et al. The stress-responsive gene GADD45G is a functional tumor suppressor, with its response to environmental stresses frequently disrupted epigenetically in multiple tumors. Clin Cancer Res 2005;11:6442-6449.

23. Qiu GH, Tan LK, Loh KS, et al. The candidate tumor suppressor gene $\mathrm{BLU}$, located at the commonly deleted region 3p21.3, is an E2Fregulated, stress-responsive gene and inactivated by both epigenetic and genetic mechanisms in nasopharyngeal carcinoma. Oncogene 2004:23:4793-4806.

24. Tao Q, Robertson KD, Manns A, et al. The Epstein-Barr virus major latent promoter $\mathrm{Qp}$ is constitutively active, hypomethylated, and methylation sensitive. J Virol 1998;72:7075-7083.

25. Wong ML, Tao Q, Fu L, et al. Aberrant promoter hypermethylation and silencing of the critical 3p21 tumor suppressor gene, RASSF1A, in Chinese oesophageal squamous cell carcinoma. Int J Oncol 2006;28:767-773.

26. Tao $Q$, Huang H, Geiman TM, et al. Defective de novo methylation of viral and cellular DNA sequences in ICF syndrome cells. Hum Mol Genet 2002;11:2091-2102.

27. Tao Q, Swinnen $L$, Yang J, et al. Methylation status of the Epstein-Barr virus major latent promoter $C$ in iatrogenic $B$ cell lymphoproliferative disease. Application of PCR-based analysis. Am J Pathol 1999;155: 619-625.

28. Korinek V, Barker N, Morin PJ, et al. Constitutive transcriptional activation by a beta-catenin-TCF complex in APC-/- colon carcinoma. Science 1997:275:1784-1787.

29. Jones PA, Baylin SB. The fundamental role of epigenetic events in cancer. Nat Rev Genet 2002;3:415-428.

30. Robertson KD, Wolffe AP. DNA methylation in health and disease. Nat Rev Genet 2000;1:11-19.

31. Rountree MR, Bachman KE, Baylin SB. DNMT1 binds HDAC2 and a new co-repressor, DMAP1, to form a complex at replication foci. Nat Genet 2000;25:269-277.

32. Chan AT, Tao Q, Robertson KD, et al. Azacitidine induces demethylation of the Epstein-Barr virus genome in tumors. J Clin Oncol 2004;22:1373-1381.

33. Szyf M, Pakneshan P, Rabbani SA. DNA demethylation and cancer: therapeutic implications. Cancer Lett 2004;211:133-143.

34. Morrison JA, Gulley ML, Pathmanathan R, et al. Differential signaling pathways are activated in the Epstein-Barr virus-associated malignancies nasopharyngeal carcinoma and Hodgkin lymphoma. Cancer Res 2004;64:5251-5260.

35. de Castro J, Gamallo C, Palacios J, et al. beta-catenin expression pattern in primary oesophageal squamous cell carcinoma. Relationship with clinicopathologic features and clinical outcome. Virchows Arch 2000;437:599-604.

36. Nakanishi $Y$, Ochiai A, Akimoto $S$, et al. Expression of E-cadherin, alphacatenin, beta-catenin and plakoglobin in esophageal carcinomas and its prognostic significance: immunohistochemical analysis of 96 lesions. Oncology 1997;54:158-165.

37. Veeramachaneni NK, Kubokura $\mathrm{H}$, Lin L, et al. Down-regulation of beta catenin inhibits the growth of esophageal carcinoma cells. J Thorac Cardiovasc Surg 2004;127:92-98. 